Antihypertensive Therapie bei Nephropathien

\title{
Schutz für Herz und Niere
}

\author{
Weltweit steigt die Prävalenz von Nierenkrankheiten. Die Hyper- \\ tonie spielt dabei eine bedeutsame Rolle im Sinne eines Circulus \\ vitiosus: Nierenerkrankungen verursachen eine - renale - Hyper- \\ tonie, und essenzieller sowie renaler Bluthochdruck schädigen \\ die Nieren. Für Patienten mit chronischer Niereninsuffizienz ist \\ das Risiko, eine schwere kardiale Erkrankung zu erleiden und \\ daran zu sterben, höher als die Wahrscheinlichkeit einer zuneh- \\ menden Nierenschädigung mit terminaler Niereninsuffizienz.
}

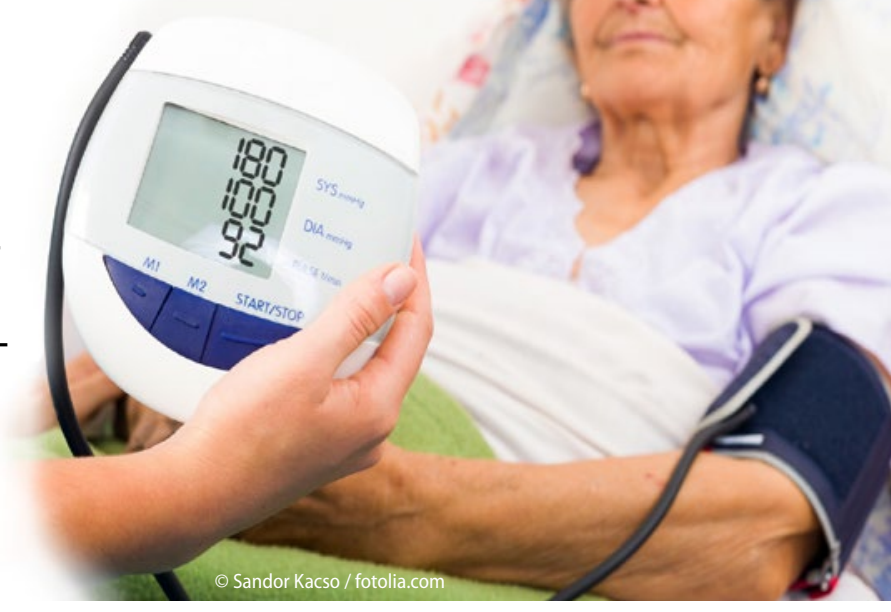

_ Die Häufigkeit von kardialen und renalen Komplikationen korreliert mit der Höhe des Blutdrucks und der Eiweißausscheidung im Urin. Prof. Dr. Michel Burnier, Lausanne (Schweiz), monierte, dass Blutdruck, Albuminurie und Nierenfunktion viel zu selten gemessen werden und dass nur eine verschwindend geringe Zahl der Patienten über ihre Nierenerkrankung informiert wird.

\section{Jeder zweite Nierenkranke verfehlt seine Blutdruckziele}

Nach den Empfehlungen der Fachgesellschaften soll bei Patienten mit chronischer Nierenerkrankung ein Blutdruck systolisch ab $140 \mathrm{mmHg}$ und diastolisch ab $90 \mathrm{mmHg}$ behandelt und unter 140 $\mathrm{mmHg}$ bzw. $85 \mathrm{mmHg}$ gesenkt werden. Dies gilt für Patienten unter 70 Jahren mit einer glomerulären Filtrationsrate (GFR) unter $60 \mathrm{ml} / \mathrm{min} / 1,73 \mathrm{~m}^{2}$ und/oder mit einer Albuminurie über $30 \mathrm{mg} / \mathrm{g}$ Kreatinin bei allen GFR-Werten. Jeder zweite Patient mit einer chronischen Nierenerkrankung erreicht die Zielwerte nicht.

ACE-Hemmer gelten seit Jahren als bevorzugte Antihypertensiva bei Patienten mit Nephropathie, weil sie nicht nur den Blutdruck, sondern auch die Albuminurie senken und die Progression der Nierenerkrankung verhindern. Hyperkaliämie und Kreatininanstieg sind Nebenwirkungen, die Kontrollen und Dosisanpassungen erfordern, nicht aber Kontraindikation für ihre Verordnung.

Für die i.d.R. nötige Kombinationstherapie stammen die wichtigsten Er- kenntnisse aus der ACCOMPLISH-Studie: Sowohl hinsichtlich schwerer kardiovaskulärer als auch renaler Komplikationen war die Kombination aus ACEHemmer und Kalziumantagonist derjenigen aus ACE-Hemmer und Diuretikum überlegen. Die ersten drei Stufen der antihypertensiven Therapie bei chronischer Nierenerkrankung sind unumstritten:

1 Hemmung des Renin-AngiotensinSystems (RAS), d.h. ACE-Hemmer oder Angiotensinrezeptorblocker

2 Kalziumantagonist (evtl. Diuretikum)

3 Diuretikum (evtl. Kalziumantagonist)

4 Betablocker, Alphablocker, fraglich: Aldosteronantagonist.

Beta- und Alphablocker senken den Blutdruck, doch fehlen Endpunktstudien bei Patienten mit Nephropathie. Für die Aldosteronantagonisten listete Burnier acht randomisierte und sieben nicht-randomisierte Studien bei diabetischer und nicht-diabetischer Nephropathie mit Spironolacton $(n=14)$ und Eplerenon $(n=1)$ auf, die zu einem Rückgang der Proteinurie um 30-50\% führten. Die gefürchtete Hyperkaliämie wurde nur in einer von acht randomisierten Studien beobachtet. Die Spironolacton-Dosis betrug in 11 Studien $25 \mathrm{mg}$. Eine sorgfältige Auswahl der Patienten und engmaschige Kontrollen von Kalium und Kreatinin sind nötig.

\section{Wirksame Therapie der} Hyperkaliämie ante portas

Einen völlig neuen Aspekt gibt es im Hinblick auf die Hyperkaliämie, die häufig die Therapie mit Hemmstoffen des Renin-Angiotensin-Systems und mit Aldosteronantagonisten limitiert. Im Jahr 2015 wurde in zwei großen Studien über erste Ergebnisse mit Patiromer, einem neuartigen Polymer, berichtet, das Kalium im Darm bindet.

In der Studie von Weir et al. (NEJM 2015) erhielten 219 Patienten mit Niereninsuffizienz Grad 3 und 4 (GFR 15$60 \mathrm{ml} / \mathrm{min} / 1,73 \mathrm{~m}^{2}$ ) und einem Serumkalium zwischen 5,1 und $6,5 \mathrm{mmol} / \mathrm{l}$ zweimal 4,2 oder 8,4 g Patiromer für 4 Wochen. Der mittlere Kaliumspiegel sank innerhalb weniger Tage von 5,6 auf 4,6 mmol/l, 76\% der Patienten erreichten den Zielwert zwischen 3,8 und 5,1 $\mathrm{mmol} / \mathrm{l}$.

In der Studie von Bakris et al. (JAMA 2015) waren es 306 Typ-2-Diabetiker mit gleicher Nierenfunktionseinschränkung (mittleres Kreatinin 1,7-2,3 mg/dl) und einem Kalium über 5,0 mEq/l. Bei leichter $(5,13 \mathrm{mEq} / \mathrm{l})$ und mittelschwerer $(5,7$ $\mathrm{mEq} / \mathrm{l})$ Hyperkaliämie kam es innerhalb von 3-4 Tagen zu einem Abfall auf 4,6 $\mathrm{mEq} / \mathrm{l}$, der 52 Wochen anhielt. In beiden Studien wurden nur leichte Nebenwirkungen beobachtet. Möglicherweise ist hier bald eine wirksame und gut verträgliche Therapie der Hyperkaliämie verfügbar, sodass RAS-Hemmer und Aldosteronantagonisten bei deutlich mehr Patienten eingesetzt werden können. -

Prof. Dr. med. Heinrich Holzgreve

- 26th Meeting, European Society of Hypertension, Paris 10.-13.6.2016 